\title{
Intercostal Artery Aneurysm in a Child with Disseminated Tuberculosis and Coexistent Takayasu Arteritis: A Rare Case Report
}

\author{
Poonam Sherwani, Mriganki Choudhary, Yash Shrivastava, Pankaj Sharma \\ Department of Radiodiagnosis, All India Institute of Medical Sciences, Rishikesh, Uttarakhand, India
}

\section{Abstract}

Intercostal (IC) artery aneurysm due to underlying tuberculosis (TB) is very rare in adults and even rarer in children, and very few cases have been reported so far. The underlying mechanism is due to erosion of the arterial wall due to adjacent inflammation such as empyema, pericarditis, or cavitatory lesion. Aortic pseudoaneurysm or Rasmussen aneurysm is common in pulmonary TB. However, asymptomatic and incidentally detected pseudoaneurysm of the right IC artery in a child with disseminated TB is infrequent. Although the association of TB and Takayasu arteritis (TA) is well known, the association of IC artery aneurysm due to underlying TB along with TA has not been reported previously.

Keywords: Intercostal artery, Takayasu arteritis, tuberculosis

\section{INTRODUCTION}

Tubercular aortoarteritis leading to mycotic aneurysm is common in developing countries due to the friable arterial wall affecting the aorta and pulmonary arteries. However, intercostal (IC) artery pseudoaneurysm due to tubercular adenitis is very rare. Trauma and interventional procedures have been commonly associated with IC aneurysm. ${ }^{[1]}$ Cross-reaction between tuberculosis (TB) and Takayasu arteritis (TA) has been reported previously in many studies. Here, we describe the case of a 15-year-old female, a follow-up case of disseminated TB, who presented with asymptomatic IC artery aneurysm secondary to an enlarged subcarinal lymph node. Imaging findings also revealed Takayasu vasculitis. As IC aneurysm was detected incidentally, medical treatment was commenced without any emergency intervention.

\section{Case Report}

A 15-year-old female presented in the emergency department with complaints of fever, abdominal pain, and vomiting for 10 days. She was diagnosed to have abdominal TB 6 months prior and was treated with antitubercular medications. However, antitubercular medicines were stopped 3 months back due to drug-induced hepatitis. General examination

\begin{tabular}{|l|l|}
\hline \multicolumn{2}{|c|}{ Access this article online } \\
\hline Quick Response Code: & Website: \\
\hline & www.ijrc.in \\
\cline { 2 - 2 } & \\
\hline
\end{tabular}

revealed pallor and discrepancy of pulses and blood pressure in both upper limbs. The pulse was feebler and blood pressure was lower in the left upper limb than the right. Blood pressure on the right upper limb was $140 / 90 \mathrm{mmHg}$, while on the left side, it was $124 / 90 \mathrm{mmHg}$. Bilateral cervical lymph nodes were also enlarged and found matted on palpation. Laboratory investigation revealed anemia and leukocytosis (hemoglobin $-8.5 \mathrm{~g} / \mathrm{dl}$ and total leukocyte count $-13,800$ cells $\left./ \mathrm{mm}^{3}\right)$. Acute phase reactants were elevated (erythrocyte sedimentation rate $-45 \mathrm{~mm} / \mathrm{h}$ and C-reactive protein $-12 \mathrm{mg} / \mathrm{L}$ ). Chest radiograph was essentially normal. Given the history of abdominal TB and discrepancy in the pulses and blood pressure in both the upper limbs, computed tomographic (CT) angiography of the thorax and abdomen was done which revealed circumferential mural thickening along with subtle irregularity involving

Address for correspondence: Dr. Poonam Sherwani, Department of Radiodiagnosis, All India Institute of Medical Sciences, Rishikesh - 249 203, Uttarakhand, India. E-mail: sherwanipoonam@gmail.com

This is an open access journal, and articles are distributed under the terms of the Creative Commons Attribution-NonCommercial-ShareAlike 4.0 License, which allows others to remix, tweak, and build upon the work non-commercially, as long as appropriate credit is given and the new creations are licensed under the identical terms.

For reprints contact: WKHLRPMedknow_reprints@wolterskluwer.com

How to cite this article: Sherwani P, Choudhary M, Shrivastava Y, Sharma P. Intercostal artery aneurysm in a child with disseminated tuberculosis and coexistent Takayasu arteritis: A rare case report. Indian J Respir Care 2021;10:129-31.

Received: 08-05-2020 Accepted: 07-08-2020

Revised: 06-08-2020 Published: 31-01-2021 
thoracic and abdominal aorta. Thickening was extending along the brachiocephalic trunk, left common carotid, and left subclavian arteries causing luminal narrowing [Figure 1a]. Diffuse mural thickening was also seen involving the aortic arch, main, and both the pulmonary arteries [Figure $1 \mathrm{~b}$ and $\mathrm{c}]$. Few collaterals were also seen in the mediastinum. There was presence of a saccular aneurysm arising from the right sixth IC artery which was seen adjacent to the necrotic lymph node in the subcarinal region [Figure 1c and d]. Diffuse mural thickening is also seen involving the abdominal aorta and along the celiac axis with severe stenosis at the origin and superior mesenteric artery [Figure $2 \mathrm{a}$ and $\mathrm{b}$ ]. The right kidney was small in size with grossly attenuated right renal artery [Figure $2 \mathrm{~b}$ ]. There was also a fusiform aneurysm of the left renal artery at the origin along with associated mural thickening [Figure 2c]. Few collaterals were also seen in the mesentery [Figure 2d]. In addition to the vascular findings, CECT chest showed presence of bilateral pleural effusion [Figure 1a], with enlarged subcarinal, and right hilar lymph nodes [Figure 3a]. Few enlarged left para-aortic lymph nodes were also present and enhancing mural thickening was seen in the distal ileal loop [Figure $3 \mathrm{~b}$ and c]. High-density ascites with omental stranding was also seen in the pelvis [Figure 3d]. Imaging findings were suggestive of disseminated TB and TB vasculitis involving the right IC artery and left renal artery. The rest of the vascular findings were likely due to TA considering the age, clinical criteria, laboratory findings, and the classical imaging findings. Hence, the final diagnosis of disseminated TB with tubercular aortoarteritis causing IC artery aneurysm and synchronous TA was made. As there was no history of hemoptysis, no interventional procedure was done for IC aneurysm and antitubercular drugs were continued along with prednisolone and methotrexate for the management of TA.

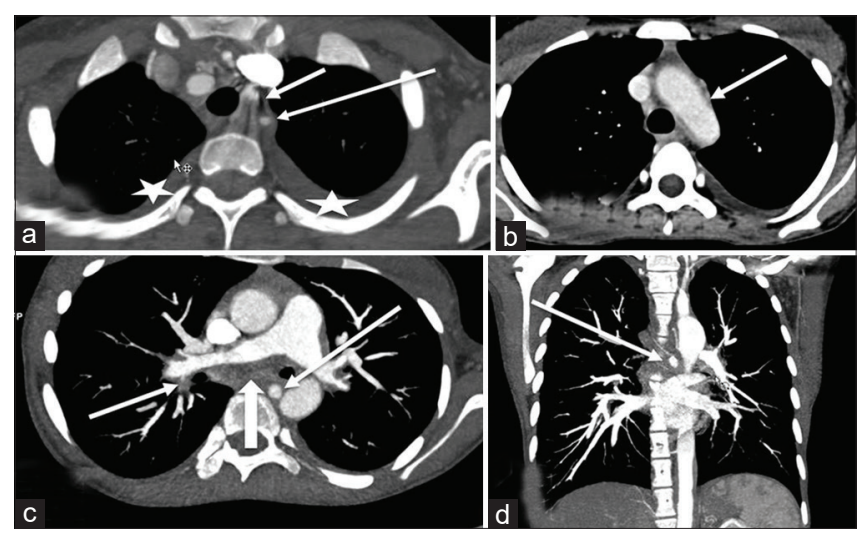

Figure 1: (a-d) Computed tomographic angiography maximum intensity projection image. Axial image depicts circumferential mural thickening of the left subclavian artery (large arrow in a), left common carotid artery (small arrow in a), aortic arch (arrow in b), and right pulmonary artery (small arrow in c). Note is also made of bilateral mild pleural effusion ( ${ }^{*}$ in a). A saccular aneurysm is seen arising from the right sixth intercostal artery in an axial image (large white arrow in c) and reconstructed coronal image (arrow in d) with a large hypodense lymph node is also seen in the subcarinal region (white block arrow)

\section{Discussion}

The association of TA and TB has been described previously in many studies, which suggest that cellular immune response targeting a group of tissue stress protein in patients with TA is similar to the host response to Mycobacterium tuberculosis. ${ }^{[2]}$

TA is grouped under large-vessel vasculitis which affects the aorta and its branches with pulmonary arteries involvement. ${ }^{[2]}$ It is also known as pulseless disease and affects adolescent girls or females $<40$ years of age. ${ }^{[2]}$ The diagnosis of TA in children is difficult as most of the children present during the inactive stage or due to sequelae of vasculitis rather than in the active stage. Hypertension is more common in children. Nonspecific symptoms such as headache, fever, and weight loss are more frequently seen in children, and the diagnosis of TA poses a clinical challenge to the clinician. ${ }^{[3]}$ Therefore, the presence of discrepancy of blood pressure and pulses in the limbs gives a clue to the diagnosis.

According to the EULAR/PRINTO/PRES Ankara 2008 classification, criteria for the diagnosis of TA in children are:

Typical findings on angiography (conventional/CT/magnetic resonance) of the aorta and pulmonary arteries include stenosis, narrowing, occlusion, or thickened aortic wall and one out of five criteria given below:

1. Pulse deficit and/or claudication induced by activity

2. Systolic blood pressure difference $>10 \mathrm{~mm}$ in bilateral upper limbs

3. Bruit or thrill over the aorta and its branches

4. Hypertension

5. Elevated acute phase reactant. ${ }^{[4]}$
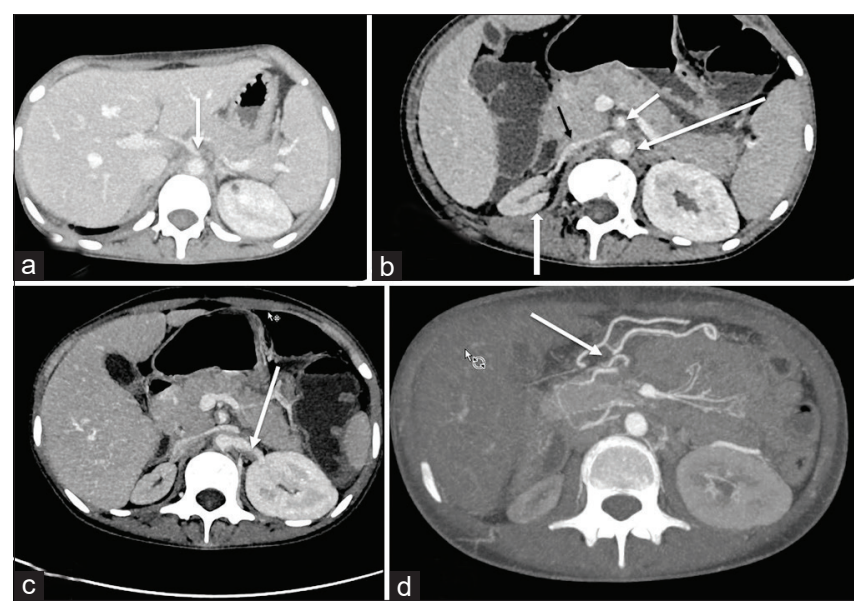

Figure 2: $(a$ and $b)$ Contrast-enhanced computed tomographic axial image depicts mural thickening at the origin of the celiac axis causing the ostial stenosis. Mural thickening is also seen involving the abdominal aorta (large arrow in b) and superior mesenteric artery (small white arrow in b). The right renal artery is grossly attenuated in caliber (black arrow in b) and small-sized right kidney (block white arrow in b). (c) Saccular aneurysm is seen in the left renal artery (arrow in c) in addition to the circumferential mural thickening. (d) Maximum intensity projection images show few collaterals in the mesentery 


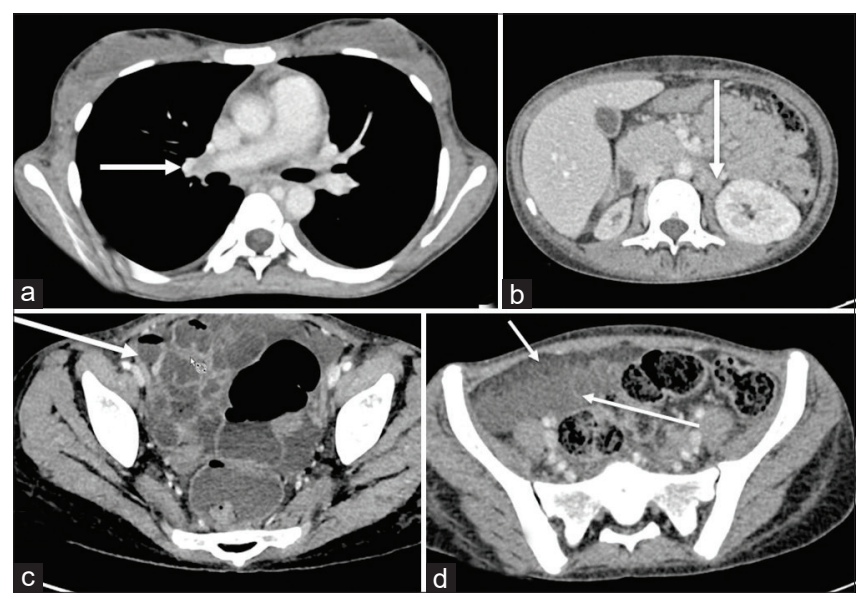

Figure 3: (a-d) Contrast-enhanced chest and abdomen axial image shows necrotic lymph node in the right hilar region (arrow in a) and conglomerated lymph nodes in the left para-aortic station (arrow in b). Enhancing mural thickening is seen in the distal ileal loop without significant luminal compromise (arrow in c). High-density ascites (large arrow in d) with omental stranding is seen in the pelvic region (small arrow in d). These findings were consistent with disseminated tuberculosis

Depending on the CT angiography and clinical and laboratory criteria, the diagnosis of TA was made in our patient. Saccular or fusiform thoracoabdominal aortic aneurysms may be associated. Renal artery stenosis is common in childhood resulting in malignant hypertension and chronic renal disease.

However, IC artery aneurysm is unusual in TA and was attributed to tubercular aortoarteritis. Tubercular aortoarteritis mimics large-vessel vasculitis and occurs mainly due to contiguous infiltration of vessels due to underlying pathology such as enlarged necrotic lymph nodes, empyema, and pericarditis in pulmonary $\mathrm{TB}$. In disseminated TB, any vessel can be affected. Contiguous involvement of the vessel wall can lead to the formation of a mycotic true or false aneurysm. ${ }^{[5]}$

Mycotic aneurysm of the IC artery in TB aortoarteritis due to underlying pathology is a very rare finding which has been rarely described before. ${ }^{[6]}$ The aneurysm of the medial right IC artery was likely due to the erosion of the wall by the subcarinal necrotic lymph nodes. Most of the patients described before were symptomatic, but our patient did not show any clinical symptoms related to IC artery aneurysm such as hemoptysis.

Management in TA in the prevascular stage includes immunosuppressive therapy with gradual tapering of dosage. ${ }^{[3]}$ Antihypertensives are used for controlling blood pressure in the vascular stage. Synchronous tubercular aortoarteritis requires the addition of antitubercular drugs. There are no established specific guidelines for the management of asymptomatic IC artery aneurysm. There was no complaint of hemoptysis in the index case, and the aneurysm was subcentimeter in size, so the child was managed conservatively. However, regular follow-up in the outpatient department was advised and to immediately report to the emergency in case of hemoptysis. Follow-up CT angiogram was also recommended after 3 months to evaluate the size of the aneurysm or any associated complications. In cases of large or ruptured or symptomatic IC aneurysm, endovascular management with coiling or glue is indicated. ${ }^{[7]}$

\section{Conclusion}

Mycotic aneurysm of the IC artery itself is very rare secondary to tuberculous infection and occurs due to inflammation in the surrounding tissues leading to either the formation of a false or true aneurysm. The association of TA and TB has been known previously. The association of tubercular aortoarteritis leading to IC aneurysm with synchronous TA in a child makes our case distinct and unique.

\section{Declaration of patient consent}

The authors certify that they have obtained all appropriate patient consent forms. In the form, the parent has given his consent for the images and other clinical information of his daughter to be reported in the journal. The parent understands that the child's name and initials will not be published and due efforts will be made to conceal her identity, but anonymity cannot be guaranteed.

\section{Financial support and sponsorship} Nil.

\section{Conflicts of interest}

There are no conflicts of interest.

\section{REFERENCES}

1. Sekino S, Takagi H, Kubota H, Kato T, Matsuno Y, Umemoto T. Intercostal artery pseudoaneurysm due to stab wound. J Vasc Surg 2005;42:352-6.

2. Pedreira AL, Santiago MB. Association between Takayasu arteritis and latent or active Mycobacterium tuberculosis infection: A systematic review. Clin Rheumatol 2019;15:1-8.

3. Brunner J, Feldman BM, Tyrrell PN, Kuemmerle-Deschner JB, Zimmerhackl LB, Gassner I, et al. Takayasu arteritis in children and adolescents. Rheumatology (Oxford) 2010;49:1806-14.

4. Ozen S, Pistorio A, Iusan SM, Bakkaloglu A, Herlin T, Brik R, et al. EULAR/PRINTO/PRES criteria for Henoch-Schönlein purpura, childhood polyarteritis nodosa, childhood Wegener granulomatosis and childhood Takayasu arteritis: Ankara 2008. Part II: Final classification criteria. Ann Rheum Dis 2010;69:798-806.

5. Goyal A, Shah I. Aortoarteritis with tuberculosis. J Family Med Prim Care 2017;6:153-4.

6. Sharma M, Singhal M, Kamble R, Bhalla A, Gorsi U, Khandelwal N. Intercostal artery pseudoaneurysm in pulmonary tuberculosis - A rare cause of hemoptysis: A case report with review of the literature. Lung India 2019;36:63-5.

7. Fenwick A, Omotoso P, Ferguson D. Endovascular management of unruptured intercostal artery aneurysms. CVIR Endovasc 2019;2:1-5. 